\title{
Total versus subtotal gastrectomy for distal gastric cancer: meta-analysis of randomized clinical trials
}

\author{
This article was published in the following Dove Press journal: \\ OncoTargets and Therapy \\ 7 November 2016 \\ Number of times this article has been viewed
}

\section{Lingling Kong* \\ Nianzhao Yang* \\ Lianghui Shi \\ Guohai Zhao \\ Minghai Wang \\ Yisheng Zhang}

Department of General Surgery, The First Affiliated Yijishan Hospital of Wannan Medical College, Wuhu, People's Republic of China

*These authors contributed equally to this work
Correspondence: Minghai Wang; Yisheng Zhang

Department of General Surgery, The First Affiliated Yijishan Hospital of

Wannan Medical College, No 92,

Zheshan Road, Wuhu, Anhui

24I00I, People's Republic of China

Tel +86553 I38 6665 8218;

+86553 I3855300038

Email wangmh0410@sina.com;

zhangys0109@hotmail.com

\begin{abstract}
Objectives: This meta-analysis of randomized controlled trials was conducted to give a more precise estimation of the efficacy and drawbacks of total gastrectomy (TG) versus subtotal gastrectomy (SG) for proven distal gastric cancer.
\end{abstract}

Methods: The electronic databases Cochrane and PubMed (updated on April 10, 2016) were searched for randomized controlled trials comparing TG with SG as surgical procedures for distal gastric cancer. Five outcome variables were analyzed, including postoperative complications, anastomotic fistula rate, hospital mortality rate, mortality rate of recurrence (the patient's death is caused by the recurrence of gastric cancer, rather than caused by other diseases), and 5-year survival rate. Random or fixed effect model was used to perform this meta-analysis.

Results: Six trials, including 573 cases treated with TG and 791 cases treated with SG, were included. Compared with patients in the SG group, patients in the TG group did not show a higher rate of postoperative complications (odds ratio [OR]: 1.46, 95\% confidence interval $[\mathrm{CI}]$ : $0.71-3.03, P=0.30)$. However, patients in the TG group showed a significantly higher rate of anastomotic fistula than patients in the SG group (OR: 3.78, 95\% CI: $1.97-7.27, P<0.0001$ ). Hospital mortality rate, which was analyzed in four trials, including 510 TG versus 729 SG patients, showed no significant difference between the two groups (OR: 1.80, 95\% CI: 0.85-3.78, $P=0.12$ ). Importantly, there was no significant difference in the 5-year survival between the two groups (OR: $0.68,95 \% \mathrm{CI}$ : $0.39-1.19, P=0.18$ ). Mortality rate of recurrence, which was also analyzed in three trials, including $396 \mathrm{TG}$ versus $407 \mathrm{SG}$ patients, showed a significantly higher rate in the TG group (OR: $0.07,95 \% \mathrm{CI}: 0.01-0.13, P=0.03$ ).

Conclusion: This meta-analysis demonstrated that postoperative complications, hospital mortality rate, and 5-year survival rate in TG patients was similar to the SG group. Furthermore, SG was associated with significantly fewer anastomotic fistula and lower mortality rate of recurrence compared with TG. However, lower mortality rate of recurrence was probably related to the criteria of these two procedures.

Keywords: total gastrectomy, subtotal gastrectomy, gastric cancer, randomized controlled trials

\section{Introduction}

Gastric cancer is one of the most common digestive tract malignancies worldwide, and surgical resection is the only therapeutic modality for cure. ${ }^{1}$ The resection method includes total gastrectomy (TG) and subtotal gastrectomy (SG). Since Billroth performed the first SG in 1881 and Schlatter the first TG in 1897, the best surgical procedure for adenocarcinoma of the distal stomach has been a subject of debate for more than a century. ${ }^{2-4}$

The routine use of TG was accepted for three main reasons: ${ }^{5-7}$ 1) TG could reduce the likelihood of recurrence due to possible inadequate lymph nodes removal; 2) it could remove all multicentric carcinoma foci in the gastric remnant; and 3) it could eliminate the risk of metachronous adenocarcinoma that may develop in the gastric remnant. 
Additionally, some studies demonstrated that TG was preferable to $\mathrm{SG}$, because patients treated with $\mathrm{TG}$ had longer 5-year survival than patients treated with $\mathrm{SG}^{8-10}$ On the other hand, advocates of SG claimed that routine use of TG increased operative morbidity and hospital mortality, and had no advantage over SG in terms of 5-year oncological results. ${ }^{11-13}$

The debate over the type of curative resection for distal gastric cancer based on a number of retrospective published studies, and patients' criteria of eligibility were different. Therefore, with respect to the trials comparing TG versus SG, well-designed randomized controlled trials (RCTs) are necessary. Until now, only a few RCTs $\mathrm{s}^{3,8,14,15}$ have reported the data on the short- and long-term outcomes of TG for distal gastric cancer in comparison to $\mathrm{SG}$, and the results reported in these RCTs were discordant.

Therefore, a more precise estimation of the outcomes of TG in comparison to SG is necessary. To our knowledge, there is no meta-analysis of the evidence gathered from the outcomes of TG and SG surgery for distal gastric cancer. This meta-analysis may give the answer in terms of the best available scientific evidence to date. The postoperative complication rate, anastomotic fistula rate, hospital mortality rate, mortality rate of recurrence, and 5-year survival rate of TG in comparison to SG were estimated in this study.

\section{Methods}

\section{Search strategy}

The electronic literature searches were conducted using Cochrane and PubMed (updated on April 10, 2016). Search terms included: "total gastrectomy", "subtotal gastrectomy", "gastric cancer", and "Randomized controlled trials". All titles, abstracts, and full-text articles were evaluated by two reviewers independently. To be eligible, the studies had to meet the following criteria: 1) RCTs of any size that investigated TG versus SG in patients with cancer in distal stomach; 2) reported on relevant short- or long-term outcomes of trial; and 3) published in peer-reviewed journals in English language.

\section{Data extraction}

The data extraction and critical appraisal from all the eligible studies was carried out independently by two investigators. The following variables were extracted from the included studies if available: first author's name, publication year, the number of patients in TG and SG groups, overall complication rate of the two groups, anastomotic fistula rate of the two groups, mortality rate of the two groups, mortality rate of recurrence of the two groups, and 5-year survival rate of the two groups. The extracted data were discussed until consensus was achieved.

\section{Statistical analysis}

The odds ratios (ORs) and 95\% confidence intervals (CIs) were used to assess all outcomes. ${ }^{13}$ We assessed the heterogeneity between studies by using the $\mathrm{Q}$ statistic, which indicates the presence of heterogeneity when $P<0.10$. If the results of studies had no heterogeneity, the fixedeffects model (the Mantel-Haenszel method) was used to calculate the pooled ORs. Otherwise, a random-effects model (the DerSimonian and Laird method) was used for meta-analysis. Statistical significance was considered when $P<0.05$. All statistical analyses were conducted using the Review Manager version 5.1.6 (Cochrane Collaboration, Oxford, UK) software package.

\section{Results}

\section{Description of eligible trials}

Six clinical trials ${ }^{8,14-18}$ published in four articles were considered suitable for this meta-analysis. The study size in two RCTs from Europe was larger than 100 patients except for the Asian trial (including 54 patients). All trials reported an appropriated method of randomization. None of the studies reported the method of blinding. Six studies contained 1,364 pooled patients, of whom 573 were allocated to the TG group, 791 to the SG group. The detailed information of each included study is shown in Table 1.

The patients were excluded from the study if: 1) curative resection could not be performed and thus conservative surgery was performed; 2) curative resection could not be performed for macroscopic lymph node involvement of the cardioesophageal junction or splenopancreatic region; 3) linitis plastica; 4) lymphoma; 5) suspected superficial carcinoma; and 6) patients with heart failure, renal insufficiency, severe diabetes or arteritis, obesity ( $>20 \%$ of normal body weight), recent myocardial infarction, and liver cirrhosis, patients who underwent laparoscopic surgery, and patients who died within 30 days after surgery. The inclusion criteria were: 1) cancer of the distal half of the stomach; 2) absence of hepatic or peritoneal spread of the tumor or metastatic deposits in the third nodal level, according to the Japanese classification; and 3) absence of unresectable infiltration of contiguous organs.

The reconstructive methods vary from trial to trial. In Gouzi's paper, TG repair was performed by a standard Roux-en-Y esophagojejunostomy. ${ }^{8}$ Reconstruction of SG was performed by a Billroth II gastrojejunostomy. Whereas, 
Table I Characteristics of included studies

\begin{tabular}{|c|c|c|c|c|c|c|}
\hline Authors & Year & Country & Patients (N) & Design & Randomization & Blinding \\
\hline \multirow[t]{2}{*}{ Gouzi et $\mathrm{al}^{8}$} & 1989 & France & SG:93 & RCT & Adequate & Not stated \\
\hline & & & TG:76 & & & \\
\hline \multirow[t]{2}{*}{ Robertson et al ${ }^{14}$} & 1994 & People's Republic & SG:25 & RCT & Adequate & Not stated \\
\hline & & of China & TG:29 & & & \\
\hline \multirow{2}{*}{ Bozzetti et al $\left.\right|^{3,15}$} & 1997, 1999 & Italy & SG:320 & RCT & Adequate & Not stated \\
\hline & & & TG:304 & & & \\
\hline \multirow[t]{2}{*}{ De Manzoni et al ${ }^{16}$} & 2003 & Italy & SG:77 & $\mathrm{RCT}$ & Adequate & Not stated \\
\hline & & & TG:40 & & & \\
\hline \multirow[t]{2}{*}{ Lee et $\mathrm{a}^{18}$} & 2010 & Korea & SG:62 & $\mathrm{RCT}$ & Adequate & Not stated \\
\hline & & & TG:63 & & & \\
\hline \multirow[t]{2}{*}{ Park et al ${ }^{17}$} & 2014 & Korea & SG:2I4 & RCT & Adequate & Not stated \\
\hline & & & TG:6I & & & \\
\hline
\end{tabular}

Abbreviations: N, numbers; RCT, randomized controlled trial; SG, subtotal gastrectomy; TG, total gastrectomy.

Robertson et $\mathrm{a}^{14}$ restored the intestinal continuity by an endto-side esophagojejunostomy, using a circular stapler, with a $40 \mathrm{~cm}$ jejunal Roux limb.

\section{Pooled efficacy of TG versus SG}

Six clinical trials containing 1,364 patients $(\mathrm{TG}=573$, $\mathrm{SG}=791$ ) were analyzed. Compared with patients in the SG group, patients in the TG group did not show a higher rate of postoperative complications (OR: 1.46, 95\% CI: $0.71-3.03, P=0.30$ ) (Figure 1). However, patients in the TG group showed a significantly higher rate of anastomotic fistula than patients in the SG group (OR: 3.78, 95\% CI: 1.97-7.27, $P<0.0001$ ) (Figure 2). Furthermore, there was no significant difference in the hospital mortality rate between the two groups (OR: $1.80,95 \% \mathrm{CI}: 0.85-3.78, P=0.12$ ) (Figure 3). Importantly, there was no significant difference in the 5-year survival between the two groups (OR: 0.68, 95\% CI: 0.39-1.19, $P=0.18$ ) (Figure 4).

Mortality rate of recurrence, which was analyzed in three trials ${ }^{14,15,18}$ that included 803 pooled patients, of whom 396 TG versus 407 SG patients, showed a significantly higher rate in the TG group (OR: $0.07,95 \% \mathrm{CI}: 0.01-0.13, P=0.03$ )
(Figure 5), with no between-study heterogeneity ( $P=0.14$, $I^{2}=49 \%$ ) (Figure 5). The detailed information of the pooled data is shown in Table 2.

\section{Discussion}

Six trials ${ }^{8,14-18}$ have been undertaken to investigate the issues of efficacy and drawbacks of TG versus SG. This metaanalysis showed that the anastomotic fistula rate and mortality rate of recurrence was higher in the TG group than the SG group. In addition, there was no difference in postoperative complication rate, hospital mortality rate, and 5-year survival rate between $\mathrm{TG}$ and $\mathrm{SG}$ groups.

Both TG and SG are surgical procedures performed with curative intent. These two surgical procedures are quite effective curative treatments, but also bring a great number of postoperative complications, which include surgical and nonsurgical complications. Two RCTs from Hong Kong ${ }^{14}$ and Italy ${ }^{15}$ revealed that the risk of postoperative complications was found to be higher in the TG group than the SG group. But RCTs from France ${ }^{8}$ and Italy $^{16}$ demonstrated that TG did not increase postoperative complications in comparison with SG. Our meta-analysis showed that there

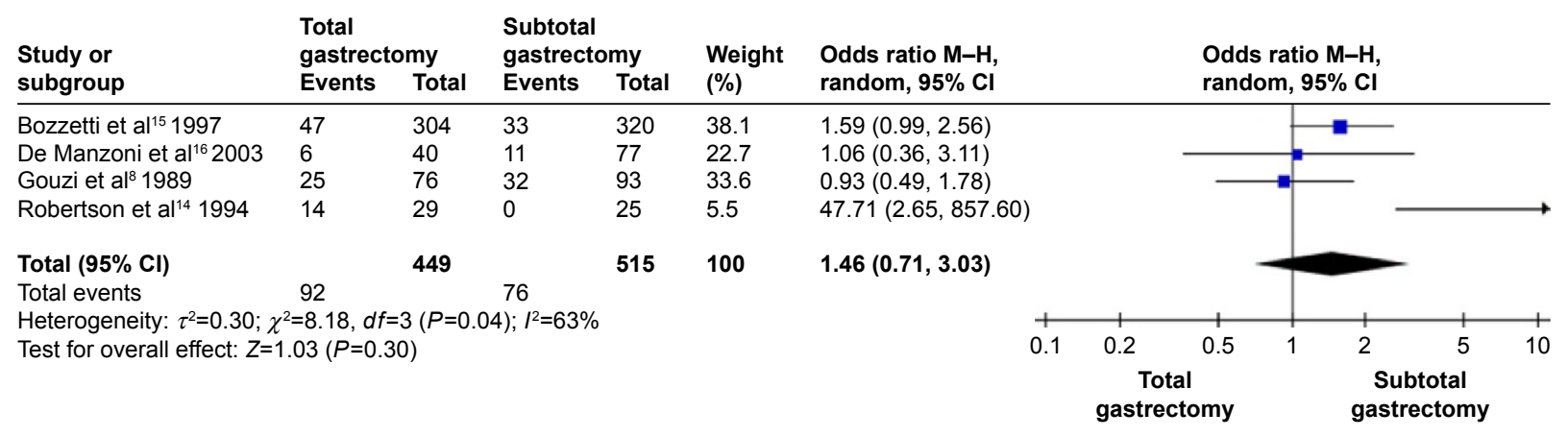

Figure I Meta-analysis of postoperative complications in randomized trials of total gastrectomy versus subtotal gastrectomy. Abbreviations: $\mathrm{Cl}$, confidence interval; $\mathrm{M}-\mathrm{H}$, Mantel-Haenszel. 


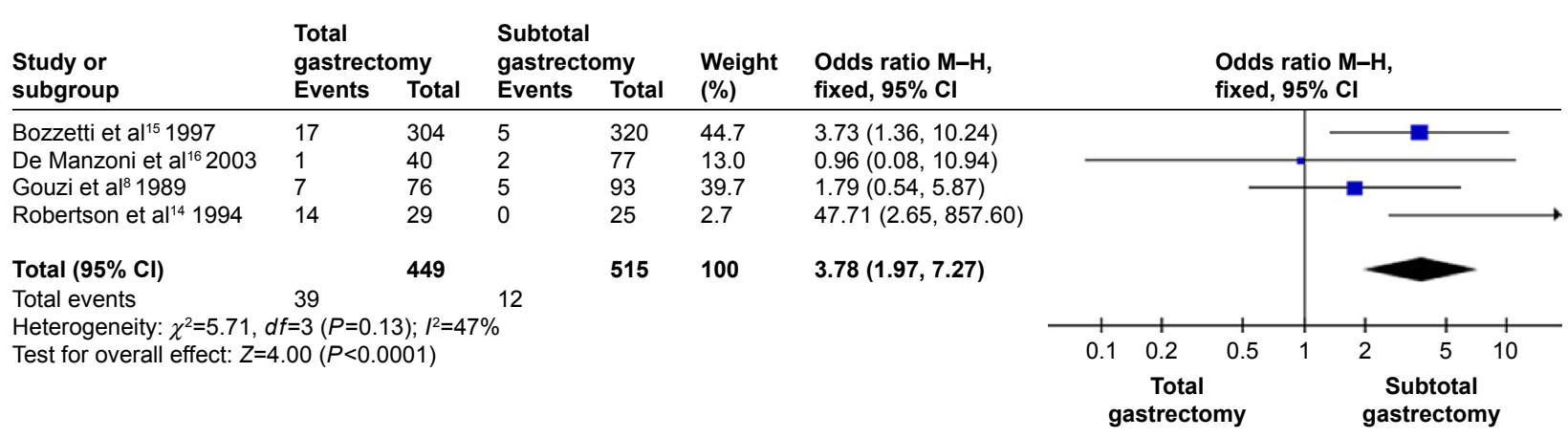

Figure 2 Meta-analysis of anastomotic fistula in randomized trials of total gastrectomy versus subtotal gastrectomy. Abbreviations: $\mathrm{Cl}$, confidence interval; $\mathrm{M}-\mathrm{H}$, Mantel-Haenszel.

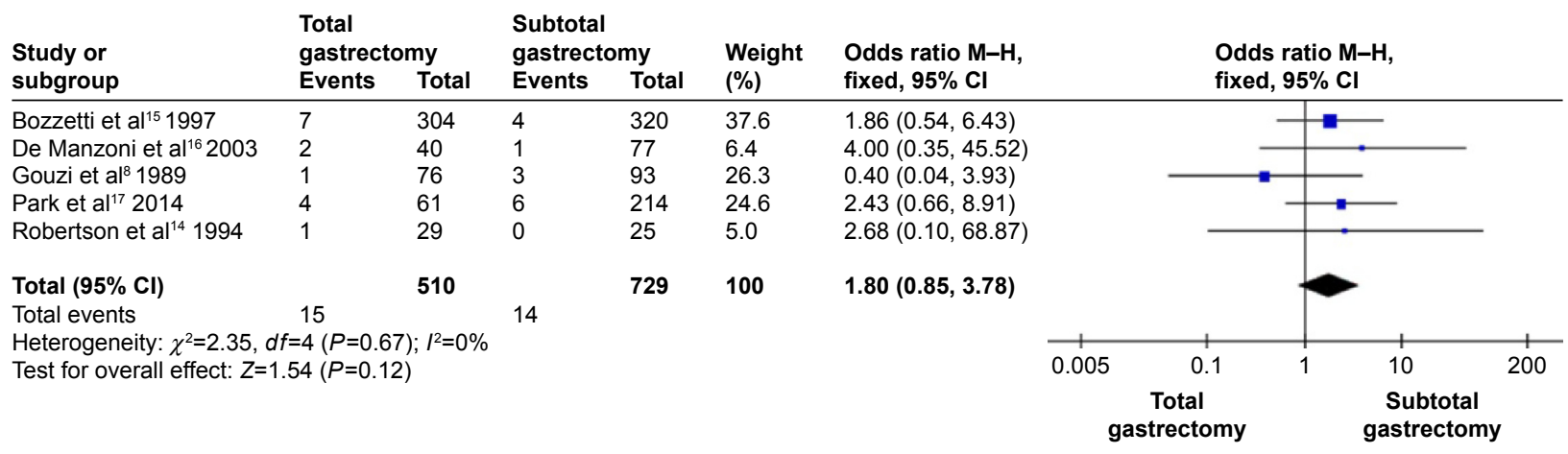

Figure 3 Meta-analysis of hospital mortality rate of total gastrectomy versus subtotal gastrectomy.

Abbreviations: $\mathrm{Cl}$, confidence interval; $\mathrm{M}-\mathrm{H}$, Mantel-Haenszel.

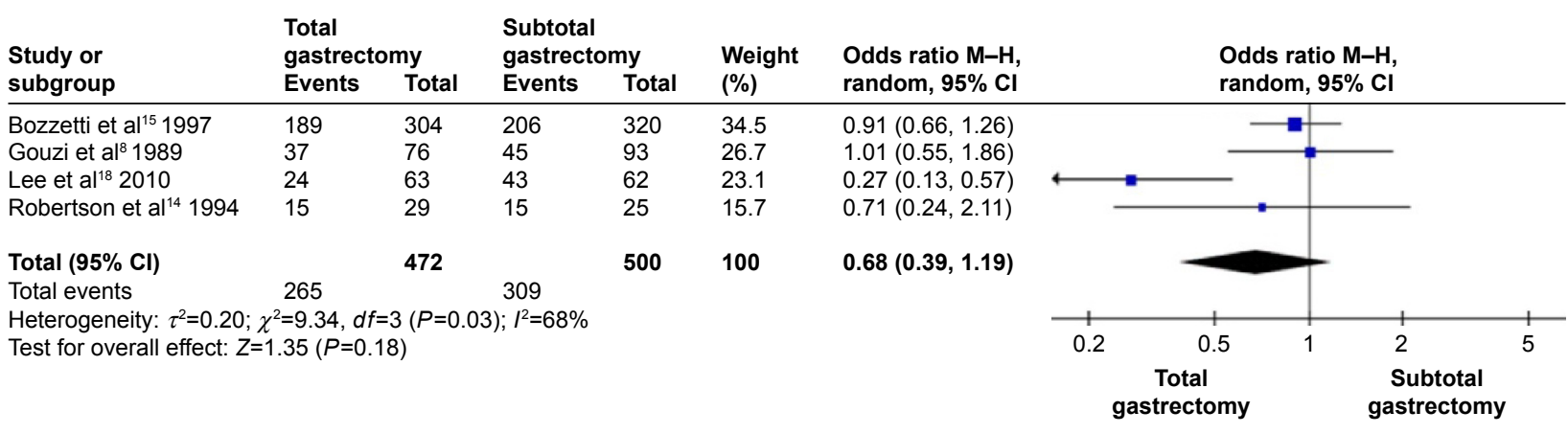

Figure 4 Meta-analysis of 5-year survival rate of total gastrectomy versus subtotal gastrectomy.

Abbreviations: $\mathrm{Cl}$, confidence interval; $\mathrm{M}-\mathrm{H}$, Mantel-Haenszel.

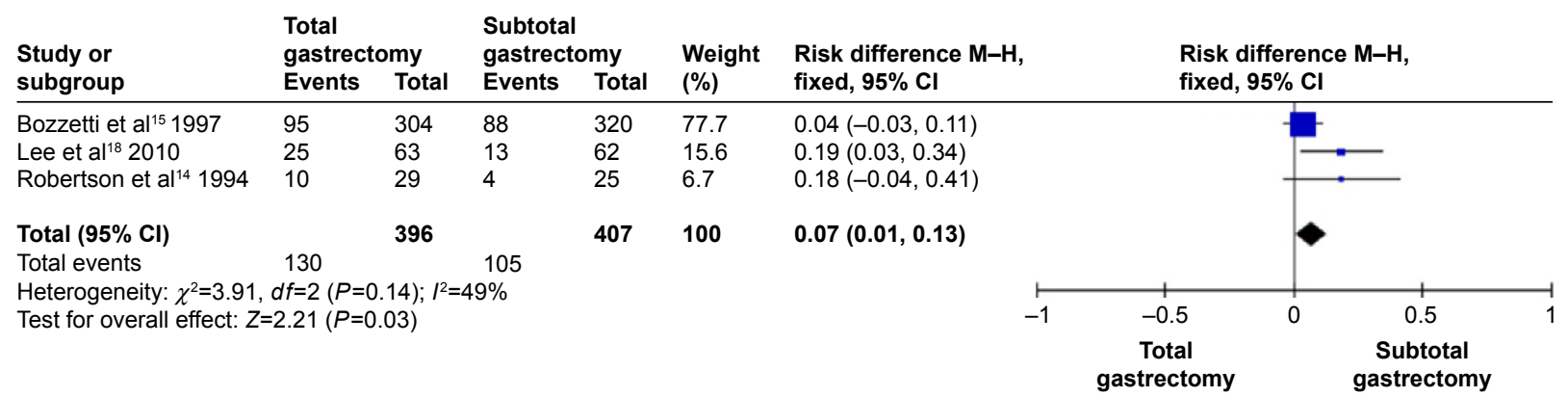

Figure 5 Meta-analysis of mortality of recurrence in randomized trials of total gastrectomy versus subtotal gastrectomy.

Abbreviations: $\mathrm{Cl}$, confidence interval; $\mathrm{M}-\mathrm{H}$, Mantel-Haenszel. 
Table 2 Summary statistics of pooled data comparing total gastrectomy versus subtotal gastrectomy

\begin{tabular}{|c|c|c|c|c|c|c|}
\hline \multirow[t]{2}{*}{ Outcome variables } & \multirow[t]{2}{*}{ OR $(95 \% \mathrm{CI})$} & \multicolumn{2}{|c|}{ Test for overall effect } & \multicolumn{3}{|c|}{ Test for heterogeneity } \\
\hline & & $\mathbf{Z}$ & $P$-value & $\mathbf{Q}$ & $P$-value & $I^{2}$ index \\
\hline Complication & $1.46(0.7 \mathrm{I}, 3.02)$ & 1.03 & 0.30 & 8.18 & 0.04 & $63 \%$ \\
\hline Anastomotic fistula & $3.78(1.97,7.27)$ & 4.00 & $<0.0001$ & 5.71 & 0.13 & $47 \%$ \\
\hline Hospital mortality & $1.80(0.85,3.78)$ & 1.54 & 0.12 & 2.35 & 0.67 & $0 \%$ \\
\hline Mortality rate of recurrence & $0.07(0.01,0.13)$ & 2.21 & 0.03 & 3.91 & 0.14 & $49 \%$ \\
\hline Five-year survival & $0.68(0.39,1.19)$ & 1.35 & 0.18 & 9.34 & 0.03 & $68 \%$ \\
\hline
\end{tabular}

Abbreviations: $\mathrm{Cl}$, confidence interval; $\mathrm{OR}$, odds ratio.

was no significant difference in postoperative complications between the TG and SG groups. The preoperative criteria of eligibility, which excluded patients in poor condition from randomization, might explain the lack of significant differences between TG and SG. ${ }^{15}$

Anastomotic fistula rate was reported in three trials. The pooled data revealed a statistically significantly higher anastomotic fistula rate in the TG group. In the Hong Kong trial led by Robertson et al, ${ }^{14}$ three anastomotic fistulae were recorded in the TG group, which were at oesophagojejunal junction. In the French trial led by Gouzi et al, ${ }^{8} 12$ anastomotic fistulae were recorded, of which 7 were in the TG group and 5 in SG group. In Italy, in a trial by De Manzoni et al, ${ }^{16}$ three anastomotic fistulae were recorded, of which one was recorded in the TG group and two in the SG group, and the total numbers of two groups were 40 and 77 , respectively. All these anastomotic fistulae were managed conservatively with nutritional support with favorable outcome. ${ }^{8,14}$ In the Italian trial led by Bozzetti et al, ${ }^{15} 22$ anastomotic fistulae were recorded, of which 17 were in the TG and 5 in the SG groups. However, this trial did not provide any information about treatment. According to Gouzi and Robertson, the procedure of TG is more complicated and time consuming. Otherwise, compared to TG, the reconstructive method of SG reflected richer blood supply to the stomach. Large clinical trials should be conducted to confirm this finding about significant difference in anastomotic fistula rate between TG and SG.

There was no significant difference of hospital mortality rate between the two groups in all the trials, although TG surgical procedure was prolonged and more complex.

All trials have revealed that there was a significantly higher mortality rate in the TG group compared with the SG group. Lower mortality rate of recurrence was probably related to the criteria of these two procedures. In addition, high mortality rate in patients undergoing resection of the stomach was usually related to anastomotic fistula. ${ }^{8}$ However, all patients who suffered from anastomotic fistula in two RCTs from Hong Kong and France were medically treated and healed without mortality ${ }^{8,14}$ Fatal complications may increase the mortality rate, but the proportion of fatal complications in the RCT from Italy was not significantly different between the TG and SG groups. ${ }^{15}$ Therefore, the pooled results showed that mortality rates in TG and SG groups were not statistically significantly different, although pooled data showed a statistically significant higher anastomotic fistula rate in the TG group compared with the SG group.

Recurrence rate was not reported in both TG and SG groups. Mortality rate of recurrence in both surgical procedures was reported in two RCTs., ${ }^{3,14}$ Our meta-analysis showed statistically significant lower mortality rate of recurrence in the SG group than the TG group. To our knowledge, the TG group has shown higher 5-year survival rate than the SG group in two retrospective studies. ${ }^{9}{ }^{10}$ However, this difference has not been observed in all trials. In contrast, the RCT from Hong Kong ${ }^{14}$ showed that overall survival was significantly better in the SG than the TG group (median survival, 1,511 vs 922 days, $P<0.05$ ). For these inconsistent conclusions, it is possible that some unknown prognostic factors were not balanced between the TG and SG groups. In the present meta-analysis, no significant difference in 5-year survival was observed between the TG and SG groups. However, even early gastric cancer was associated with a high frequency of second primaries. ${ }^{19}$ Therefore Bozzetti et $\mathrm{a}^{3}{ }^{3,15}$ suggested that with respect to the procedure of choice for distal gastric cancer, TG should not be rejected in principle.

On the other hand, there are several limitations in this meta-analysis. First, limited number of patients with cancer of the middle one-third of the stomach were randomized to either a TG or a SG group, although a majority of patients in the Italian trial had cancer of the antrum. Second, the SG group with D1 lymphadenectomy was not in line with the TG group with D2 lymphadenectomy in the Hong Kong trial. Third, the inclusion of studies published only in English is another potential limitation of this analysis. Fourth, only two trials mentioned the reconstructive method, so it is hard to assess whether or not the reconstructive method influences the prognosis. Finally, only four RCTs were conducted in the 1990s in some countries, and the sample is small. Further well-designed randomized clinical trials with larger sample size are still needed to get a more 
precise estimation of the efficacy and drawbacks of TG versus SG for distal gastric cancer.

In conclusion, the present study suggested that postoperative complication rate, mortality rate, and 5-year survival rate in the TG group were similar to that in the SG group. Furthermore, SG was associated with significantly less anastomotic fistula and lower mortality rate of recurrence compared with TG. Therefore, this study demonstrated that TG is not superior to SG. Further well-designed randomized clinical trials with larger sample size are still needed to get a more precise estimation of the short- and long-term results of TG for distal gastric cancer in comparison to SG.

\section{Acknowledgments}

This project was sponsored by the grants from National Science Foundation of China (81372828) and Scientific Research Fund Project of Introduction of Talents of The Yijishan Hospital Affiliated to Wannan Medical College (YR201305).

\section{Author contributions}

Conception and design: MHW and YSZ. Furthermore, MHW takes full responsibility for the integrity of the work as a whole, from inception to published article. Acquisition, compilation, and interpretation of the data: NZY and LLK; LHS and GHZ were responsible for statistical analysis of this article, including writing $\mathrm{R}$ codes, interpretation of the results, and computer output of the data. All authors were involved in drafting the manuscript and revising it critically for important intellectual content and have given final approval for the version to be published. Furthermore, all authors have participated sufficiently in the work to take public responsibility for its content.

\section{Disclosure}

The authors report no conflicts of interest in this work.

\section{References}

1. Kim MC, Kim KH, Jung GJ. A 5 year analysis of readmissions after radical subtotal gastrectomy for early gastric cancer. Ann Surg Oncol. 2012;19(8):2459-2464.

OncoTargets and Therapy

\section{Publish your work in this journal}

OncoTargets and Therapy is an international, peer-reviewed, open access journal focusing on the pathological basis of all cancers, potential targets for therapy and treatment protocols employed to improve the management of cancer patients. The journal also focuses on the impact of management programs and new therapeutic agents and protocols on
2. Egli S, Schlatter K, Streule R, Lage D. A structure-based expert model of the ICD-10 mental disorders. Psychopathology. 2006;39(1):1-9.

3. Bozzetti F, Marubini E, Bonfanti G, Miceli R, Piano C, Gennari L. Subtotal versus total gastrectomy for gastric cancer: five-year survival rates in a multicenter randomized Italian trial. Italian Gastrointestinal Tumor Study Group. Ann Surg. 1999;230(2):170-178.

4. Billroth T. [General surgical pathology and therapy. Guidance for students and physicians. Lecture]. Khirurgiia (Mosk). 1991;(10):136-143. Russian.

5. Yamamoto M, Yamanaka T, Baba H, Kakeji Y, Maehara Y. The postoperative recurrence and the occurrence of second primary carcinomas in patients with early gastric carcinoma. J Surg Oncol. 2008;97(3):231-235.

6. Wu B, Wu D, Wang M, Wang G. Recurrence in patients following curative resection of early gastric carcinoma. J Surg Oncol. 2008;98(6):411-414.

7. Braga M, Molinari M, Zuliani W, et al. Surgical treatment of gastric adenocarcinoma: impact on survival and quality of life. A prospective ten year study. Hepatogastroenterology. 1996;43(7):187-193.

8. Gouzi JL, Huguier M, Fagniez PL, et al. Total versus subtotal gastrectomy for adenocarcinoma of the gastric antrum. A French prospective controlled study. Ann Surg. 1989;209(2):162-166.

9. Lortat-Jacob J, Giuli R, Estenne B, Clot P. [Value of total gastrectomy for treatment of cancers of the stomach. Study of 482 radical operations]. Chirurgie. 1975;101(1):59-67. French.

10. McNeer G, Bowden L, Booner RJ, McPeak CJ. Elective total gastrectomy for cancer of the stomach: end results. Ann Surg. 1974;180(2):252-256.

11. Roukos D, Schmidt-Mathiesen A, Encke A. Adenocarcinoma of the gastric antrum: does D2 total gastrectomy with splenectomy improve prognosis compared to D1 subtotal gastrectomy? A long-term survival analysis with emphasis on Lauren classification. Surg Oncol. 1995;4(6): 323-332.

12. Gockel I, Pietzka S, Gonner U, Hommel G, Junginger T. Subtotal or total gastrectomy for gastric cancer: impact of the surgical procedure on morbidity and prognosis - analysis of a 10-year experience. Langenbecks Arch Surg. 2005;390(2):148-155.

13. Bradburn MJ, Deeks JJ, Berlin JA, Russell Localio A. Much ado about nothing: a comparison of the performance of meta-analytical methods with rare events. Stat Med. 2007;26(1):53-77.

14. Robertson CS, Chung SC, Woods SD, et al. A prospective randomized trial comparing R1 subtotal gastrectomy with R3 total gastrectomy for antral cancer. Ann Surg. 1994;220(2):176-182.

15. Bozzetti F, Marubini E, Bonfanti G, et al. Total versus subtotal gastrectomy: surgical morbidity and mortality rates in a multicenter Italian randomized trial. The Italian Gastrointestinal Tumor Study Group. Ann Surg. 1997;226(5):613-620.

16. De Manzoni G, Verlato G, Roviello F, et al. Subtotal versus total gastrectomy for T3 adenocarcinoma of the antrum. Gastric Cancer. 2003;6(4): 237-242.

17. Park S, Chung HY, Lee SS, Kwon O, Yu W. Serial comparisons of quality of life after distal subtotal or total gastrectomy: what are the rational approaches for quality of life management? J Gastric Cancer. 2014;14(1): $32-38$.

18. Lee JH, Kim YI. Which is the optimal extent of resection in middle third gastric cancer between total gastrectomy and subtotal gastrectomy? J Gastric Cancer. 2010;10(4):226-233.

19. Bozzetti F. Comment on "Subtotal versus total gastrectomy for T3 adenocarcinoma of the antrum". Gastric Cancer. 2004;7(3):183-184.

\section{Dovepress}

patient perspectives such as quality of life, adherence and satisfaction. The manuscript management system is completely online and includes a very quick and fair peer-review system, which is all easy to use. Visit http://www.dovepress.com/testimonials.php to read real quotes from published authors. 